\title{
Antitumor activity of a potent MEK inhibitor, TAK-733, against colorectal cancer cell lines and patient derived xenografts
}

\author{
Christopher H. Lieu ${ }^{1}$, Peter J. Klauck ${ }^{1}$, Patrick K. Henthorn ${ }^{1}$, John J. Tentler ${ }^{1}$, Aik- \\ Choon Tan ${ }^{1}$, Anna Spreafico ${ }^{1}$, Heather M. Selby ${ }^{1}$, Blair C. Britt ${ }^{1}$, Stacey M. Bagby ${ }^{1}$, \\ John J. Arcaroli ${ }^{1}$, Wells A. Messersmith ${ }^{1}$, Todd M. Pitts ${ }^{1}$ and S. Gail Eckhardt ${ }^{1}$ \\ ${ }^{1}$ Department of Medicine, Division of Medical Oncology, University of Colorado Anschutz Medical Campus, Aurora, CO, USA \\ Correspondence to: Christopher H. Lieu, email: Christopher.Lieu@ucdenver.edu \\ Keywords: MEK, colorectal cancer, patient derived xenografts, TAK-733 \\ Received: August 09, $2015 \quad$ Accepted: September 05, $2015 \quad$ Published: October 01, 2015
}

This is an open-access article distributed under the terms of the Creative Commons Attribution License, which permits unrestricted use, distribution, and reproduction in any medium, provided the original author and source are credited.

\section{ABSTRACT}

Background: CRC is a significant cause of cancer mortality, and new therapies are needed for patients with advanced disease. TAK-733 is a highly potent and selective investigational novel MEK allosteric site inhibitor.

Materials and Methods: In a preclinical study of TAK-733, a panel of CRC cell lines were exposed to varying concentrations of the agent for $\mathbf{7 2}$ hours followed by a sulforhodamine B assay. Twenty patient-derived colorectal cancer xenografts were then treated with TAK-733 in vivo. Tumor growth inhibition index (TGII) was assessed to evaluate the sensitivity of the CRC explants to TAK-733 while linear regression was utilized to investigate the predictive effects of genotype on the TGII of explants.

Results: Fifty-four CRC cell lines were exposed to TAK-733, while 42 cell lines were deemed sensitive across a broad range of mutations. Eighty-two percent of the cell lines within the sensitive subset were BRAF or KRAS/NRAS mutant, whereas $80 \%$ of the cell lines within the sensitive subset were PIK3CA WT. Twenty patientderived human tumor CRC explants were then treated with TAK-733. In total, 15 primary human tumor explants were found to be sensitive to TAK-733 (TGII $\leq 20 \%$ ), including 9 primary human tumor explants that exhibited tumor regression (TGII > $100 \%$ ). Explants with a BRAF/KRAS/NRAS mutant and PIK3CA wild-type genotype demonstrated increased sensitivity to TAK-733 with a median TGII of $-6 \%$. MEKresponse gene signatures also correlated with responsiveness to TAK-733 in KRASmutant CRC.

Conclusions: The MEK inhibitor TAK-733 demonstrated robust antitumor activity against CRC cell lines and patient-derived tumor explants. While the preclinical activity observed in this study was considerable, single-agent efficacy in the clinic has been limited in CRC, supporting the use of these models in an iterative manner to elucidate resistance mechanisms that can guide rational combination strategies.

\section{INTRODUCTION}

The RAS-RAF-MEK-ERK (MAPK) pathway is a major contributor to cell growth and survival and is frequently dysregulated in numerous cancers $[6,9]$. Signaling through the MAPK pathway is known to be complex with numerous downstream effector signaling pathways and can be initiated by several growth factor receptors, including the epidermal growth factor receptor
(EGFR). Once the receptor is activated, it serves to activate membrane bound RAS which can then recruit RAF to the membrane. RAS thus serves as a critical link between growth factor receptors and initiation of signal transduction. RAS proteins are comprised of 4 major forms, HRAS, NRAS, and 2 forms of KRAS while RAF includes three kinase family members, ARAF, BRAF, and CRAF. The complexity of RAF activation is increased by additional non-RAS signaling activities 
including phosphorylation (p21 activated kinase) and dephosphorylation (protein phosphatase 2A) that are required to fully activate RAF function. RAF function is also regulated by interactions with other proteins including 14-3-3 proteins and heat shock protein 90 (Hsp90) [1]. RAF activation leads to its binding with a scaffoldlike protein complex in the cytoplasm that allows it to physically locate near the vicinity of MEK1/2. MEK1 and 2 have only one known substrate, ERK [2], whereas ERK1 and ERK 2 are known to have over 160 different targets including cytosolic proteins and numerous transcription factors [3]. The RAS/RAF/MEK/ERK cascade is a central signaling pathway required for normal cellular proliferation and transformation, and MEK has been shown to be integral in the development and progression of colorectal cancer [4].

Due to the frequent aberration of this signaling cascade in malignant tissues, MEK has emerged as an attractive target in cancer. Inhibition of MEK impairs proliferation and affects a diverse array of cellular events including differentiation, apoptosis, and angiogenesis [5, 6]. MEK is a validated target in several malignancies, including non-small lung cancer and melanoma with selumetinib and trametinib respectively [7, 8]. MEK inhibitors have also shown promise in preclinical studies of KRAS/NRAS/BRAF mutant colorectal cancer, but early phase clinical trials with the MEK inhibitor selumetinib (AZD6244 hydrogen sulfate) failed to demonstrate significant improvement in progression-free survival [9, $10]$.

MEK1/2 (MAP2K1/K2), the canonical targets of MEK inhibitors, are dual-specificity threonine/tyrosine kinases that are integral in the activation of the RAS/ $\mathrm{RAF} / \mathrm{MEK} / \mathrm{ERK}$ pathway and are often upregulated in a variety of tumor cell types. TAK-733 is a highly potent and selective novel MEK allosteric site inhibitor with an $\mathrm{IC}_{50}$ of $3.2 \mathrm{nM}$ that selectively binds to and inhibits the activity of MEK1/2, preventing the activation of MEK1/2dependent effector proteins and transcription factors. TAK-733 has demonstrated potent anticancer activity in several solid tumor mouse xenograft models and exhibited potent enzymatic and cell activity with an $\mathrm{EC}_{50}$ of $1.9 \mathrm{nM}$ against ERK phosphorylation, the downstream target within the RAS/RAF/MEK/ERK pathway, in cells [11, 12].

In this study, the antitumor activity of TAK-733 was assessed against colorectal cancer cell lines and patientderived tumor xenografts (PDX). Given the known resistance mechanisms of $\mathrm{MEK}$ inhibition in colorectal cancer, we hypothesized that tumors with known KRAS/ NRAS or BRAF mutations that were PIK3CA wild-type would exhibit greater sensitivity to MEK inhibition [13].

\section{RESULTS}

\section{Effects of TAK-733 on proliferation of colorectal cancer cell lines}

Initially, a panel of 54 CRC cell lines was exposed to TAK-733 to establish the $\mathrm{IC}_{50} \mathrm{~s}$. As depicted in Figure 1, 54 CRC cell lines were segregated into highly sensitive $\left(\mathrm{IC}_{50} \leq 0.03 \mu \mathrm{M}\right)$ or highly resistant $\left(\mathrm{IC}_{50}>1 \mu \mathrm{M}\right)$. Of the 54 cell lines, 42 (77\%) were classified as sensitive to TAK733. Cell lines with a KRAS/NRAS or BRAF mutation were associated with sensitivity to TAK-733 $(p=0.03)$, and even greater sensitivity was observed in 14 of 17 CRC cell lines that were KRAS/NRAS mutant and PIK3CA wild-type.

\section{Effects of TAK-733 on CRC cell lines by immunoblotting}

The effects of TAK-733 on the modulation of downstream targets in the MAPK and PI3K pathways were analyzed in 2 sensitive and 4 resistant cell lines (Figure 2). As observed by us and others in prior studies, inhibition of p-ERK was observed in both $\mathrm{S}$ and $\mathrm{R}$ cell lines, with only one of the R cell lines (LS123) exhibiting inhibition at only the higher $(1.25 \mathrm{uM})$ concentration [14-16]. Interestingly, there was evidence of increased p-AKT after exposure to TAK-733 in one of the R cell lines (Colo741). Two additional TAK-733 R cell lines were evaluated, and one $\mathrm{R}$ cell line was found to have an increase in p-AKT (LS123) (Supplemental Figure 1). An increase in p-AKT was also observed in one of the $\mathrm{S}$ cell lines (LOVO).

\section{MEK pathway inhibition by TAK-733 in patient- derived CRC xenografts}

To further investigate this agent, we conducted in vivo experiments in patient-derived CRC xenograft models (PDX). Based on our in vitro results, we assessed the ability of this molecular classifier: BRAF MUT (any PI3K), or KRAS/NRAS MUT and PI3K WT to predict responsiveness to TAK-733 in 20 CRC PDXs (Figure 3). To do this, we selected more clinically relevant criteria for categorizing the PDX as "responsive" or "resistant", requiring the tumor growth inhibition index to be $\leq$ $20 \%$ to score a PDX as "responsive" while a TGII > $20 \%$ was classified as "resistant". Table 1 depicts the mutational status of the PDX. Overall, there was a 75\% TGII "response rate" with 15 responders using the criteria described above. There was a trend towards greater TGII in PDXs that were KRAS/BRAF mutant and PIK3CA wild-type. Notably, of the $12 \mathrm{KRAS} / \mathrm{NRAS} / \mathrm{BRAF}$ mutant 


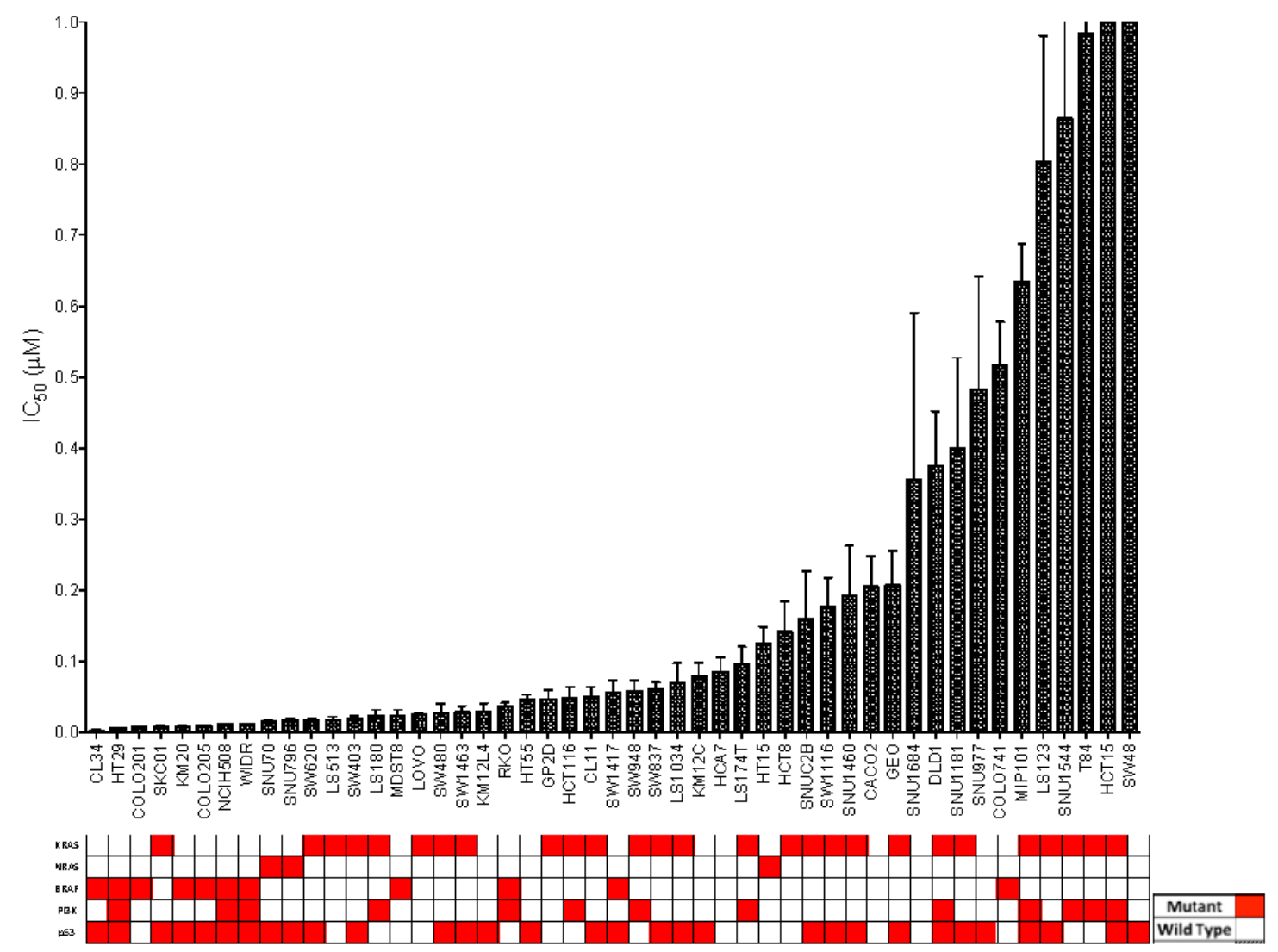

Figure 1: CRC cell lines exposed to TAK-733 to establish their IC50s. Cell lines with an IC50 of $>0.5 \mu \mathrm{M}$ are considered to be resistant. There was a broad range of sensitivity to the agent. Mutant genes are shown in red. Eighty-two percent of sensitive cell lines were BRAF or KRAS mutant $(p=0.03)$.

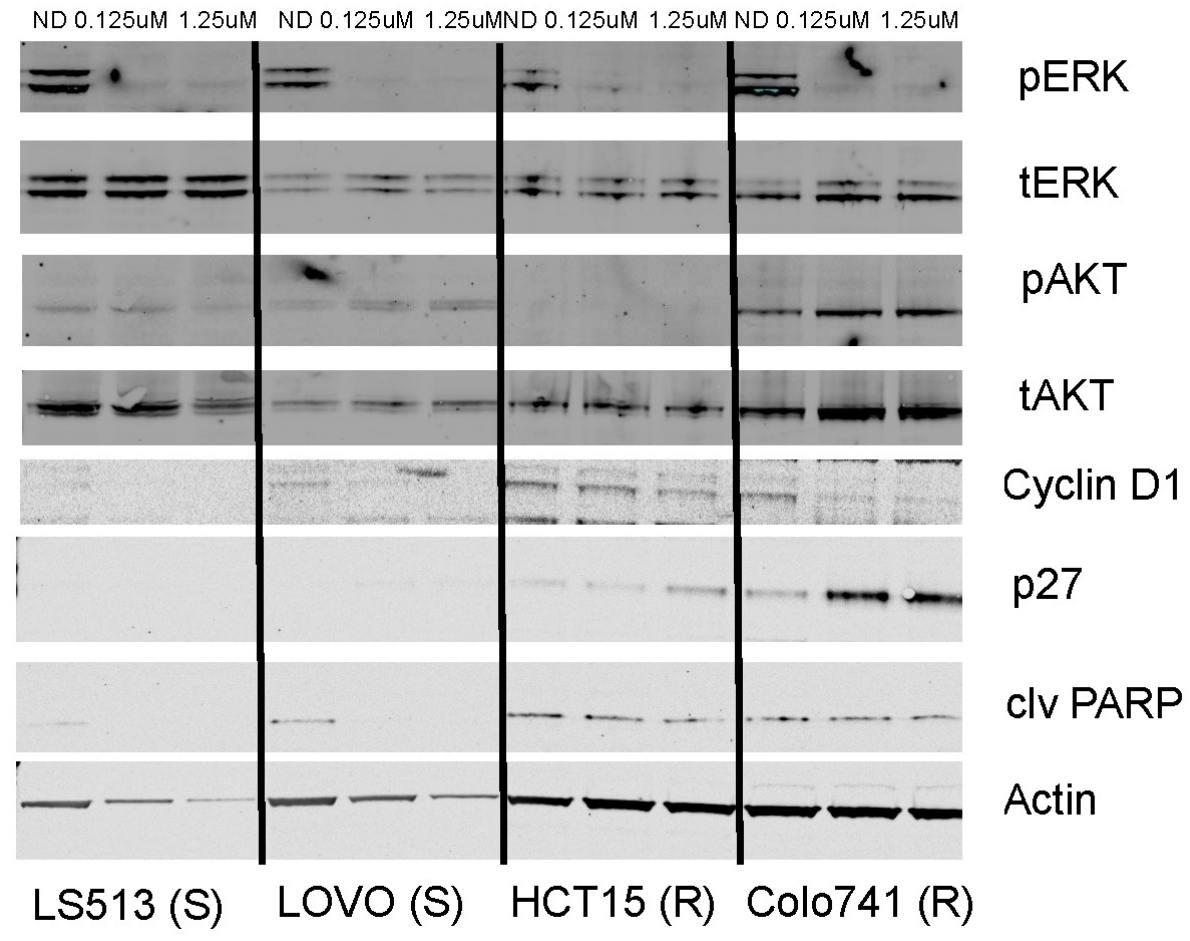

Figure 2: Effect of TAK-733 on downstream effectors. Two sensitive and resistant CRC cell lines were exposed to TAK-733. S and $\mathrm{R}$ represent sensitivity or resistance to TAK-733. 
Table 1: Mutational status of all treated PDX

\begin{tabular}{|c|c|c|c|c|}
\hline PDX & KRAS & NRAS & PIK3CA & BRAF \\
\hline 001 & MUT (G12D) & WT & WT & WT \\
\hline 007 & MUT (G13D) & WT & MUT (3’UTR) & WT \\
\hline 010 & WT & WT & WT & WT \\
\hline 012 & MUT (G12V) & WT & WT & WT \\
\hline 026 & WT & MUT & WT & WT \\
\hline 034 & WT & WT & WT & WT \\
\hline 036 & MUT (G12A) & WT & WT & WT \\
\hline 040 & MUT (G12V) & WT & MUT (543) & WT \\
\hline 042 & MUT (G13D) & WT & MUT (3'UTR) & WT \\
\hline 047 & WT & MUT (Q61K) & WT & WT \\
\hline 052 & MUT (G12V) & WT & WT & WT \\
\hline 098 & MUT (G13D) & WT & MUT (E542K) & WT \\
\hline 099 & WT & WT & WT & WT \\
\hline 102 & MUT (G12V) & WT & WT & WT \\
\hline 106 & WT & WT & WT & WT \\
\hline 108 & MUT (G12C) & WT & WT & WT \\
\hline 114 & WT & WT & WT & MUT (V600E) \\
\hline 125 & WT & WT & WT & WT \\
\hline 138 & MUT (G12D) & WT & WT & WT \\
\hline 166 & WT & WT & WT & WT \\
\hline
\end{tabular}

and PIK3CA wild-type tumors, 8 (67\%) exhibited stable disease or partial response per TGII criteria. Interestingly, of the 8 PDXs that demonstrated tumor regression, 6 (75\%) were KRAS/BRAF mutant and PIK3CA wild-type, whereas the other 2 were either all wild-type, or all mutant for RAS/RAF or PI3KCA.

\section{Pharmacodynamic markers of MEK pathway inhibition with TAK-733}

Analyses of downstream effector modulation at the end of study in 2 sensitive and 2 resistant PDX models treated with TAK-733 are depicted in Figure 4. As observed in the cell lines, suppression of p-ERK was observed in all tumors independent of responsiveness, although one could argue there was a more robust effect in the most sensitive tumor, CUCRC114, with a TGII of
$-67 \%$ that was accompanied by a reduction in p-AKT. The other biomarkers assessed were quite variable such as survivin, which was paradoxically decreased in the two non-responsive tumors and increased in one of the sensitive tumors, perhaps confounded by its assessment at the end of study. Next tumor samples from a responsive PDX (CUCRC 102) collected at the end of study were evaluated by immunohistochemistry (IHC). As depicted in Figure 5, after treatment with TAK-733, this PDX demonstrated the expected decrease in p-ERK with an increase in caspase 3. Similar results were observed in IHC stains performed on CUCRC102. 
Table 2: KRAS mutant CRC cell lines sensitivity across four MEK inhibitors. Note: nd = not determined.

\begin{tabular}{|c|c|c|c|c|}
\hline CRC Cell Lines & TAK733 (This Paper) & $\begin{array}{c}\text { AZD6244 (Tentler } \\
\text { et al MCT2010) }\end{array}$ & $\begin{array}{c}\text { PD-901 } \\
\text { (Pitts et al } \\
\text { PLoS ONE2014) }\end{array}$ & $\begin{array}{c}\text { U0126 (Flanigan et al } \\
\text { CCR2013) }\end{array}$ \\
\hline LOVO & $\mathrm{S}$ & $\mathrm{S}$ & $\mathrm{S}$ & $\mathrm{nd}$ \\
\hline SKCO1 & $\mathrm{S}$ & $\mathrm{S}$ & $\mathrm{nd}$ & $\mathrm{nd}$ \\
\hline LS513 & $\mathrm{S}$ & $\mathrm{S}$ & $\mathrm{nd}$ & $\mathrm{S}$ \\
\hline SW403 & $\mathrm{S}$ & $\mathrm{S}$ & $\mathrm{nd}$ & $\mathrm{nd}$ \\
\hline LS1034 & $\mathrm{S}$ & $\mathrm{S}$ & $\mathrm{S}$ & $\mathrm{nd}$ \\
\hline SW620 & $\mathrm{S}$ & $\mathrm{S}$ & $\mathrm{nd}$ & $\mathrm{S}$ \\
\hline LS123 & $\mathrm{R}$ & $\mathrm{R}$ & $\mathrm{R}$ & $\mathrm{R}$ \\
\hline HCT15 & $\mathrm{nd}$ & $\mathrm{R}$ & $\mathrm{R}$ & $\mathrm{R}$ \\
\hline $\mathrm{DLD} 1$ & $\mathrm{R}$ & $\mathrm{nd}$ & $\mathrm{R}$ & $\mathrm{nd}$ \\
\hline GP2D & $\mathrm{R}$ & $\mathrm{nd}$ & $\mathrm{R}$ & $\mathrm{nd}$ \\
\hline T84 & $\mathrm{R}$ & $\mathrm{nd}$ & $\mathrm{nd}$ & $\mathrm{nd}$ \\
\hline
\end{tabular}

Table 3: Prediction of the MEK inhibitor sensitivity signature and KRAS-dependency signature in KRAS mutant CRC PDX models treated with TAK-733.

\begin{tabular}{|c|c|c|c|c|}
\hline Explants & \begin{tabular}{|l} 
TAK-733 \\
Response \\
(TGII \%) \\
\end{tabular} & \begin{tabular}{|cr} 
TAK-733 & Response \\
$(\mathrm{TGII}<20 \%=\mathrm{S} ;$ TGII \\
$>20 \%=\mathrm{R})$
\end{tabular} & $\begin{array}{c}\text { MEKi Signature } \\
\text { (This Paper) }\end{array}$ & \begin{tabular}{|l|}
$\begin{array}{l}\text { KRAS-dependency } \\
\text { Signature (Singh et al Cell } \\
\text { 2012) }\end{array}$ \\
\end{tabular} \\
\hline CUCRC012 & -14 & $S$ & $\mathrm{~S}$ & IND \\
\hline CUCRC001 & -8 & $\mathrm{~S}$ & $\mathrm{~S}$ & DEP \\
\hline CUCRC102 & -6 & $\mathrm{~S}$ & $\mathrm{R}$ & DEP \\
\hline CUCRC040 & -4 & $\mathrm{~S}$ & $\mathrm{~S}$ & IND \\
\hline CUCRC052 & -4 & $\mathrm{~S}$ & $\mathrm{R}$ & IND \\
\hline CUCRC098 & 1 & $S$ & $S$ & DEP \\
\hline CUCRC108 & 7 & $\mathrm{~S}$ & $S$ & DEP \\
\hline CUCRC138 & 18 & $\mathrm{~S}$ & $\mathrm{~S}$ & DEP \\
\hline CUCRC007 & 29 & $\mathrm{R}$ & $\mathrm{S}$ & DEP \\
\hline CUCRC042 & 36 & $\mathrm{R}$ & $\mathrm{R}$ & IND \\
\hline CUCRC036 & 39 & $\mathrm{R}$ & $\mathrm{R}$ & IND \\
\hline
\end{tabular}

Table 4: Prediction of the MEK inhibitor sensitivity signature in KRAS wildtype or BRAF mutant CRC PDX models treated with TAK-733.

\begin{tabular}{|l|l|l|l|}
\hline Explants & $\begin{array}{l}\text { TAK-733 Response } \\
\text { (TGII \%) }\end{array}$ & $\begin{array}{l}\text { TAK-733 Response } \\
\text { (TGII 20\%= } \\
\text { TGII }>\mathbf{2 0 \%}=\mathbf{R} \text { ) }\end{array}$ & $\begin{array}{l}\text { MEKi Signature } \\
\text { (This Paper) }\end{array}$ \\
\hline CUCRC034 & -33 & $\mathrm{~S}$ & $\mathrm{R}$ \\
\hline $\begin{array}{l}\text { CUCRC114 (BRAF } \\
\text { mut) }\end{array}$ & -31 & $\mathrm{~S}$ & $\mathrm{R}$ \\
\hline CUCRC026 & -1 & $\mathrm{~S}$ & $\mathrm{~S}$ \\
\hline CUCRC166 & 1 & $\mathrm{~S}$ & $\mathrm{R}$ \\
\hline CUCRC125 & 4 & $\mathrm{~S}$ & $\mathrm{R}$ \\
\hline CUCRC106 & 12 & $\mathrm{~S}$ & $\mathrm{R}$ \\
\hline CUCRC010 & 17 & $\mathrm{~S}$ & $\mathrm{~S}$ \\
\hline CUCRC099 & 24 & $\mathrm{R}$ & $\mathrm{R}$ \\
\hline CUCRC047 & 84 & $\mathrm{R}$ & $\mathrm{R}$ \\
\hline
\end{tabular}




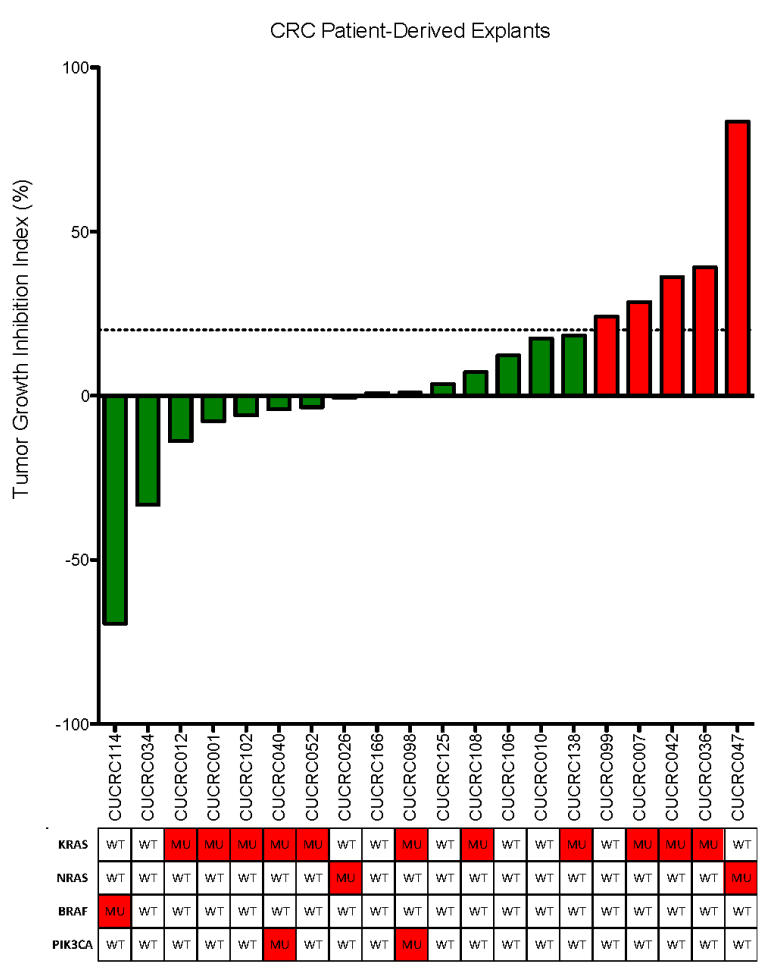

Figure 3: Tumor growth inhibition index (TGII) of all explants: TGII = treated over control, thus lower numbers indicate greater tumor reduction. Fifteen explants were found to be sensitive to TAK-733. KRAS/BRAF mutant and PIK3CA wildtype demonstrated increased sensitivity to TAK-733.
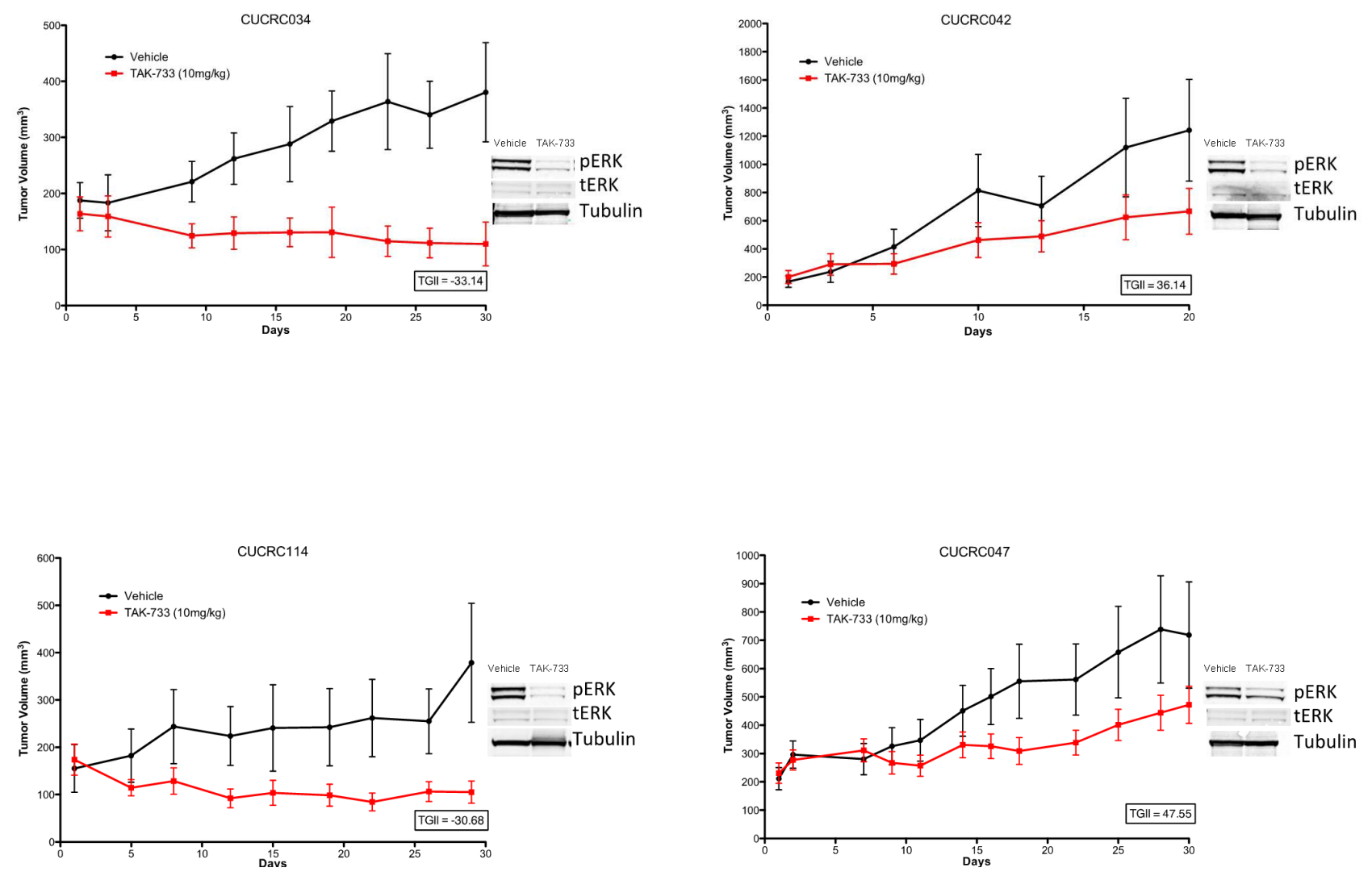

Figure 4: Individual growth curves of 2 sensitive and 2 resistant CRC patient-derived tumor explants (PDX) showing decreases in pERK in TAK-733 treated explants. 


\section{Development of a MEK-sensitivity signature for KRAS mutant CRC}

As KRAS mutation is a negative predictor for EGFR-based therapy for CRC patients, novel therapeutics are urgently needed for this population. Recent studies have suggested that the KRAS-mutant cancer cell lines, including CRC could be divided into two groups based on their "KRAS-dependency" [17, 18]. Based on the observation that the presence of a KRAS mutation was associated with sensitivity to a wide-range of MEK inhibitors including TAK-733 in CRC, but was insufficient for prediction alone, we reasoned that gene expression changes within this subset might enable better prediction of responsiveness to MEK inhibition. To test this, we focused on 11 KRAS mutant CRC cell lines that have been tested in our laboratory and demonstrated consistent sensitivity or resistance across four different MEK inhibitors [TAK-733, AZD6244 [19], PD-0325901 [20] and U0126 [21]] (Table 2). Using SAM analysis, we identified 201 probe sets that were differentially expressed in MEK inhibitor sensitive or resistant cell lines. To test whether these probe sets were predictive in KRAS mutant PDX models, we performed cluster analysis on the common 117 probe sets found between the cell lines and PDX models from two different platforms. From the

A.

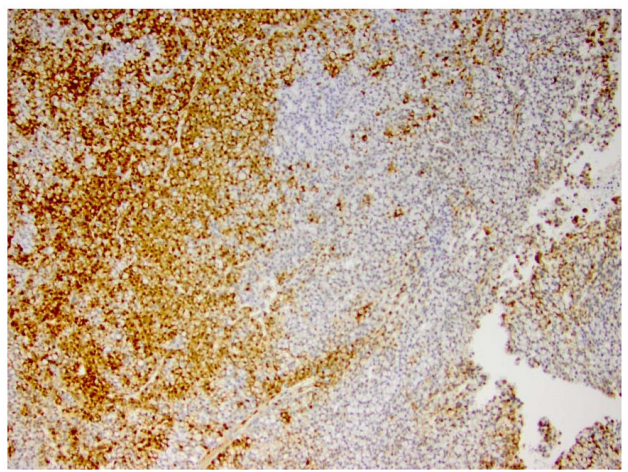

C.

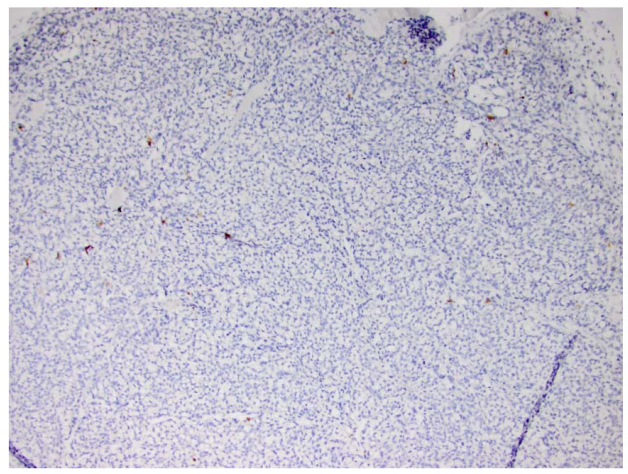

11 KRAS mutant PDX models treated with TAK-733, 7 and 4 models were predicted as sensitive or resistant, respectively (Table 3). The MEK signature correctly predicted 8 out of the 11 PDX models (accuracy 73\%) in TAK-733 sensitivity. The sensitivity and specificity of the MEK signature to TAK-733 are $86 \%(6 / 8)$ and $67 \%$ $(2 / 3)$, respectively. We also tested the published KRASdependency signature against these 11 PDX models, and this signature achieved $64 \%$ accuracy in predicting TAK733 sensitivity. The KRAS-dependency signature has $83 \%$ and $40 \%$ for sensitivity and specificity, respectively. When we tested the MEK signature against the eight KRAS wild-type and one BRAF mutant PDX models treated with TAK-733, the prediction accuracy was only $44 \%$, suggesting that the MEK signature is only predictive for KRAS mutant CRC (Table 4).

\section{DISCUSSION}

TAK-733 is a highly potent and selective novel MEK allosteric site inhibitor and selectively binds to and inhibits the activity of MEK $1 / 2$. The purpose of this study was to evaluate the antitumor activity of TAK-733 in colorectal cancer cell lines and PDX models. We also developed a MEK-sensitivity signature based on four different MEK inhibitors from colorectal cancer cell lines and evaluated the predictivity of this signature in TAK-

B.

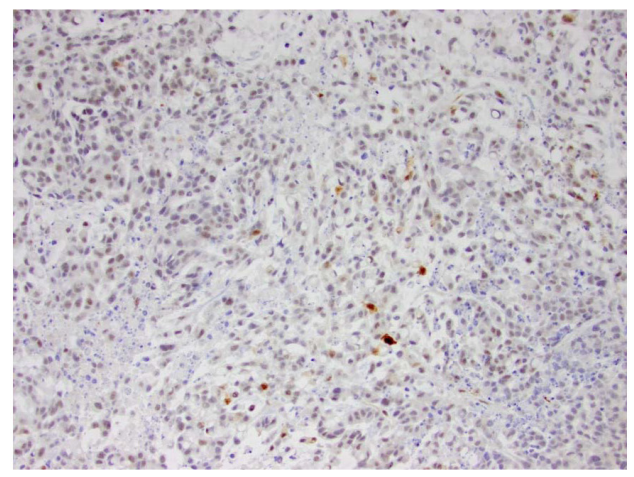

D.

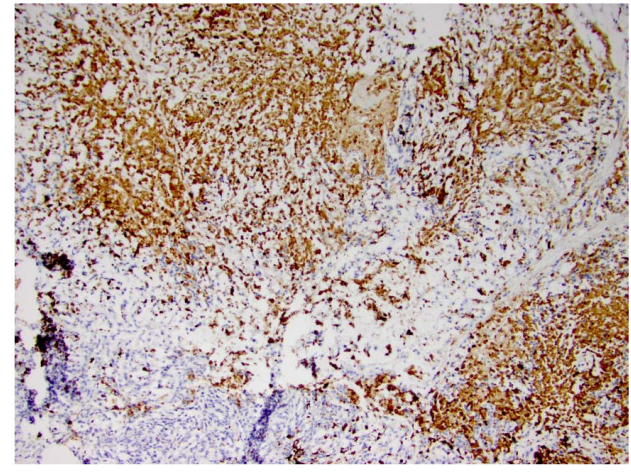

Figure 5: Representative IHC stains of p-ERK in A) control and B) treated PDXs (top) and caspase 3 in control and treated PDXs (bottom). 
733 sensitivity in PDX models. As the results demonstrate, TAK-733 exhibited significant activity against colorectal cancer cell lines and PDX models, supporting inhibition of this pathway as a therapeutic strategy in CRC, with the caveat that better prediction is needed for single agent use, or the development of rational combinations.

Prior clinical studies with an earlier generation MEK inhibitor, selumetinib, failed to demonstrate significant improvements in progression-free survival in CRC [10]. In terms of preclinical activity, TAK-733 differs from selumetinib in its potency and ability to inhibit MEK1/2 activity with an $\mathrm{IC}_{50}$ for MEK1/2 of $3.6 \mathrm{nM}$ compared to that of selumetinib of $14 \mathrm{nM}$ [22]. In a prior study of selumetinib, roughly half of the cell lines exposed to selumetinib had $\mathrm{IC}_{50}$ values $>1 \mu \mathrm{M}$, whereas in the current study, 42 of $54 \mathrm{CRC}$ cell lines exhibited robust sensitivity to TAK-733, as defined by an $\mathrm{IC}_{50} \leq 0.02 \mu \mathrm{M}$, with the majority being KRAS or BRAF mutant. Similar to studies of other MEK inhibitors, phospho-ERK was consistently suppressed to varying degrees and did not correlate with sensitivity. Likewise, other downstream effectors were variably impacted by TAK-733 and no clear conclusions could be made with respect to resistance mechanisms, although further studies are planned. These and other data suggest that at least in $\mathrm{CRC}$, various inherent and adaptive resistance pathways exist to $\mathrm{MEK}$ inhibition that will require rational strategies for combination therapy $[14$, $15,21,23]$.

In order to provide a more clinically relevant preclinical platform for in vivo testing, we utilized patientderived xenograft (PDX) models which may be better at recapitulating the tumor heterogeneity observed in patients in terms of gene-expression patterns, mutational status, and tumor architecture [24]. Additionally, we utilized more stringent response criteria to TAK-733 by setting a cutoff of TGII $<20$, similar to RECIST measurements utilized in the clinical trial setting [25]. In our study, TAK733 demonstrated substantial activity with 9 of 20 PDXs exhibiting tumor regression. This is unusual for a MEK inhibitor in CRC, and of published preclinical studies of selumetinib and trametinib, we could only find 3 models where regression was induced as a single agent, and this was largely in cell line xenograft models [14, 26-28]. Furthermore, there was a trend towards tumors displaying regression in KRAS/BRAF mutant and PIK3CA wildtype status, suggesting a potential hypothesis that can be tested in future preclinical studies of TAK-733. Similar to what was observed in cell lines, downregulation of p-ERK was consistently observed regardless of response and it was difficult to ascertain the contribution of other potential resistance pathways at the end of study, although robust caspase 3 induction was observed in a model with significant regression. While prior studies of MEK inhibition in CRC and melanoma have indicated resistance through PI3K pathway activation, our results were not consistent across multiple models, and may in fact, reflect the fact that at least in the case of CRC, combinations of PI3K and MEK inhibitors have not been particularly active in the clinic [29, 30]. As has been reported by us and others, resistance to MEK inhibition in CRC is multifactorial and related to secondary mutation events, feedback loops, or compensatory pathway activation, all of which require improved detection methodology so that combination therapy can be individualized $[15,31]$.

Not surprisingly, there was significant activity of TAK-733 against KRAS mutant CRC, due to constitutively active MEK and ERK phosphorylation in this subset. Structural and functional analyses have indicated that MEK inhibitors with superior anti-tumor activity in KRAS-driven tumors form a strong hydrogenbond interaction with the backbone amide of S212 in MEK that is critical for blocking MEK feedback phosphorylation by wild-type RAF [32]. The pyridine oxygen within the structure of TAK-733 is able to form a hydrogen bond as described above, and this interaction coupled with the potent inhibition of phosphorylated MEK may explain the anti-tumor activity observed in KRAS and BRAF mutant models, whereas activity in the non-mutant models continues to be a mystery that warrants further study.

From the MEK inhibitor signature, KRAS and $S P R Y 2$ were among the highly expressed genes in the sensitive group. Both genes are regulators of the MAPK signaling pathway and thus the sensitive lines are "dependent" on this pathway. Among the genes highly expressed in the MEK signature that predict resistance to this class of inhibitors are FZD2, a biomarker that we previously described as modulating resistant to AZD6244 via non-canonical Wnt pathway [15, 19]. We further demonstrated the combination of AZD6244 and Cyclosporin A (calcineurin inhibitor) is synergistic in KRAS mutant CRC PDXs [15]. This combination has been translated into a Phase I/II clinical trial (ClinicalTrials.gov ID: NCT02188264) at our institute. Another highly expressed gene in the MEK signature in the resistant group is anti-apoptotic gene $B C L 2 L 12$, a BCL2-family member. A recent synthetic lethality screen of MEK inhibitor (AZD6244) in KRAS mutant cancer identifies the anti-apoptotic gene BCL-XL as the top hit. The combination of ABT-263 (navitoclax, a chemical inhibitor of the BCL2 family) and a MEK inhibitor shows synergistic effects in KRAS mutant CRC xenografts [23], and this combination is currently being tested in a Phase I/II clinical trial (ClinicalTrials.gov ID: NCT02079740). Taken together, the MEK inhibitor sensitivity signature is biologically relevant and provides a list of candidate resistant genes for future combination studies with MEK inhibitors.

In summary, TAK-733 is a potent and selective MEK allosteric site inhibitor demonstrating significant activity against CRC cell lines and PDXs with KRAS and BRAF mutations. In particular, some CRC PDX models 
exhibited significant tumor regression, particularly those harboring mutations in KRAS and BRAF with no mutation in PIK3CA. This activity in CRC provides a rationale for further clinical study in patients with advanced CRC with a potential patient-selective biomarker strategy focusing on KRAS and BRAF mutant, PIK3CA wild-type tumors. Further studies will need to focus on elucidating mechanisms of resistance to TAK-733 and strategies to overcome resistance pathways with novel combination therapies.

\section{MATERIALS AND METHODS}

\section{Cell culture and proliferation analysis}

All human colon cancer cells were grown in RPMI medium supplemented with $10 \%$ fetal bovine serum (FBS), 1\% nonessential amino acids, and 1\% penicillin/ streptomycin and were maintained at $37^{\circ} \mathrm{C}$ in an incubator under an atmosphere containing 5\% $\mathrm{CO}_{2}$. The cells were routinely screened for the presence of mycoplasma (MycoAlert; Cambrex Bio Science) and were exposed to TAK-733 when they reached approximately $70 \%$ confluence. All cell lines were tested and authenticated by the University of Colorado Cancer Center DNA Sequencing and Analysis Core. DNA from CRC cell lines was analyzed using the Profiler Plus Kit (Applied Biosystems).

The antiproliferative effects of TAK-733 against CRC cell lines were determined using the sulforhodamine B (SRB) method. Briefly, cells in logarithmic growth phase were transferred to 96-well flat-bottomed plates with lids. Cell suspensions $(100 \mu \mathrm{L})$ containing 3,000 to 5,000 viable cells were plated into each well and incubated overnight before exposure with increasing concentrations of TAK-733 for 72 hours. After treatment, medium was removed and the cells were fixed with cold $10 \%$ TCA for 30 minutes at $4{ }^{\circ} \mathrm{C}$. The cells were then washed with water and stained with $0.4 \%$ SRB (Fisher Scientific) for 30 minutes at room temperature and washed again with $1 \%$ acetic acid followed by stain solubilization with 10 $\mathrm{mmol} / \mathrm{L}$ of Tris at room temperature. The plate was then read on a 96-well plate reader (Biotek Synergy 2) set at an absorbance wavelength of $565 \mathrm{~nm}$. Cell proliferation curves were derived from the raw absorbance data and expressed as the percentage of vehicle-treated controls.

\section{Immunoblotting analysis}

Cells were initially plated into 6-well plates and cultured in RPMI with 10\% FBS for 24 hours. All cells were then cultured in serum-free RPMI medium for 16 hours to lower the basal levels of ERK and AKT phosphorylation. The cells were treated with vehicle or
TAK-733 for 2 hours and then exposed to $10 \%$ FBS or serum-free media for 10 minutes. After treatment, the cells were immediately disrupted in RIPA lysis buffer containing protease and phosphatase inhibitors (50 $\mathrm{mmol} / \mathrm{L}$ of Tris-HCL, $\mathrm{pH} 7.4,150 \mathrm{mmol} / \mathrm{L}$ of $\mathrm{NaCl}$, $1 \mathrm{mmol} / \mathrm{L}$ of PMSF, $1 \mathrm{mmol} / \mathrm{L}$ EDTA, $5 \mu \mathrm{g} / \mathrm{mL}$ of aprotonin, $5 \mu \mathrm{g} / \mathrm{mL}$ of leupeptin, $1 \%$ Triton X-100, 1\% sodium deoxycholate, and $0.1 \%$ sodium dodecyl sulfate). Forty micrograms of total protein was loaded onto a $10 \%$ polyacrylamide gel, electrophoresed, and then transferred to nitrocellulose using the G2 Fast Blotter (Pierce). Membranes were blocked for 1 hour in blocking buffer $[0.1 \%$ casein solution in $0.2 \times$ phosphate buffered saline (PBS)] and were then incubated overnight at $4^{\circ} \mathrm{C}$ in blocking buffer plus $0.1 \%$ Tween-20 with the primary antibodies (Cell Signaling). Blots were then washed $3 \times$ 10 minutes in $1 \times$ TBS containing $0.1 \%$ Tween-20 and incubated with the appropriate secondary goat anti-rabbit and goat anti-mouse $\operatorname{IgG}(\mathrm{H}+\mathrm{L})$ DyLight $^{\mathrm{TM}}$ conjugated antibodies at 1:15,000 (Cell Signaling) for 1 hour at room temperature. Following $3 \times 10$ minutes of washes, the blots were developed using the Odyssey Infrared Imaging System (LI-COR Biosciences). Immunoblot experiments were done in triplicate for each antibody and representative blots are depicted.

\section{Patient-derived tumor explant models}

PDX establishment and treatment protocols were conducted under previously described procedures [33, 34]. Briefly, surgical specimens from patients undergoing either removal of a primary CRC or metastatic tumor at the University of Colorado Hospital were reimplanted s.c. into five mice for each patient. Tumor samples were then passaged into subsequent generations of mice for drug studies. Briefly, tumors were allowed to grow to a size of 1,000 to $1,500 \mathrm{~mm}^{3}$ (F1) at which point they were harvested, divided, and transplanted to an additional five mice (F2) to maintain the tumor bank. After a subsequent growth passage, tumors were excised, transplanted onto both flanks of nude mice, and expanded into cohorts of $\geq 25$ mice for treatment. Tumors from this cohort were allowed to grow until reaching approximately 150 to $300 \mathrm{~mm}^{3}$, at which time they were equally distributed by size into the two treatment groups: control and TAK733 treated. Because of the variability in take rates of the human patient explant material, enough mice were designated into each group based on the number of overall tumors ( $n=$ at least 12 tumors per group). Mice were treated for at least 28 days with either vehicle control $(0.5 \%$ methylcellulose $)$ or TAK-733 $(1 \mathrm{mg} / \mathrm{kg})$ once daily by oral gavage. Tumor growth inhibition index was calculated from average volume of the treated $(V)$ and vehicle control $\left(V_{\mathrm{vc}}\right)$ groups, with the equation: TGII $=100 \times\left(V_{\text {t final }}-V_{\text {tinitial }}\right) /\left(V_{\mathrm{vc} \mathrm{final}}-V_{\mathrm{vc} \mathrm{intial}}\right)$. 


\section{Gene expression analysis and the development of a MEK sensitivity signature}

Raw microarray gene expression data for the KRAS mutant CRC cell lines was obtained from the Cancer Cell Lines Encyclopedia (GSE36133). These samples were profiled by the Affymetrix HG-U133 Plus 2 arrays. Gene expression profiles were normalized by RMA and extracted using Affymetrix Power Tools (APT). Significant Analysis of Microarrays (SAM) (PMID: 11309499) was performed using R, with 500 permutations. Probe sets that passed FDR $<0.25$ were selected as significantly differentially expressed in MEK sensitive and resistant KRAS mutant CRC cell lines. Raw microarray gene expression data for the CRC PDX models were profiled by the Affymetrix HuGene 1.0 arrays. Gene expression profiles were normalized by RMA and extracted using APT. Probe sets were matched between platforms using the BEST_MATCH probe sets provided by Affymetrix. Probe sets that matched between two platforms were Z-normalized independently and merged into single gene expression profiles for cluster analysis. We used Spearman's rank correlation with Average linkage analysis in Cluster 3.0 (PMID: 14871861) and visualized in Java TreeView for the cluster analysis.

\section{GRANT SUPPORT}

Financial support for these studies provided by the Conquer Cancer Foundation Career Development Award (AWD-130730) and the National Institutes of Health (NIH) grant K12CA086913-11 to C.H.L. Studies were also supported by Takeda Pharmaceuticals to S.G.E., Department of Defense IDEA Award W81XWH-11-1-0526 to S.G.E., Department of Defense IDEA Award W81XWH-11-1-0527 to A.C.T., and the University of Colorado Cancer Center Grant P30 CA046934.

\section{CONFLICTS OF INTEREST}

The authors declare no conflict of interest.

\section{REFERENCES}

1. Roberts PJ and Der CJ. Targeting the Raf-MEK-ERK mitogen-activated protein kinase cascade for the treatment of cancer. Oncogene. 2007; 26:3291-3310.

2. Duffy A and Kummar S. Targeting mitogen-activated protein kinase kinase (MEK) in solid tumors. Targeted oncology. 2009; 4:267-273.

3. Britten CD. PI3K and MEK inhibitor combinations: examining the evidence in selected tumor types. Cancer chemotherapy and pharmacology. 2013; 71:1395-1409.
4. Montagut $\mathrm{C}$ and Settleman J. Targeting the RAF-MEKERK pathway in cancer therapy. Cancer Letters. 2009; 283:125-134.

5. Sebolt-Leopold JS and Herrera R. Targeting the mitogenactivated protein kinase cascade to treat cancer. Nat Rev Cancer. 2004; 4:937-947.

6. Roberts PJ and Der CJ. Targeting the Raf-MEK-ERK mitogen-activated protein kinase cascade for the treatment of cancer. Oncogene. 2007; 26:3291-3310.

7. Jänne PA, Shaw AT, Pereira JR, Jeannin G, Vansteenkiste J, Barrios C, Franke FA, Grinsted L, Zazulina V, Smith P, Smith I and Crinò L. Selumetinib plus docetaxel for KRASmutant advanced non-small-cell lung cancer: a randomised, multicentre, placebo-controlled, phase 2 study. The Lancet Oncology. 2013; 14:38-47.

8. Flaherty KT, Robert C, Hersey P, Nathan P, Garbe C, Milhem M, Demidov LV, Hassel JC, Rutkowski P, Mohr P, Dummer R, Trefzer U, Larkin JMG, Utikal J, Dreno B, Nyakas M, et al. Improved Survival with MEK Inhibition in BRAF-Mutated Melanoma. New England Journal of Medicine. 2012; 367:107-114.

9. Yeh JJ, Routh ED, Rubinas T, Peacock J, Martin TD, Shen XJ, Sandler RS, Kim HJ, Keku TO and Der CJ. KRAS/BRAF mutation status and ERK1/2 activation as biomarkers for MEK1/2 inhibitor therapy in colorectal cancer. Molecular Cancer Therapeutics. 2009; 8:834-843.

10. Bennouna J, Lang I, Valladares-Ayerbes M, Boer K, Adenis A, Escudero P, Kim T-Y, Pover G, Morris C and Douillard J-Y. A Phase II, open-label, randomised study to assess the efficacy and safety of the MEK1/2 inhibitor AZD6244 (ARRY-142886) versus capecitabine monotherapy in patients with colorectal cancer who have failed one or two prior chemotherapeutic regimens. Investigational New Drugs. 2011; 29:1021-1028.

11. Dong Q, Dougan DR, Gong X, Halkowycz P, Jin B, Kanouni T, O'Connell SM, Scorah N, Shi L, Wallace MB and Zhou F. Discovery of TAK-733, a potent and selective MEK allosteric site inhibitor for the treatment of cancer. Bioorganic \&amp; Medicinal Chemistry Letters. 2011; 21:1315-1319.

12. Wallace MB, Adams ME, Kanouni T, Mol CD, Dougan DR, Feher VA, O'Connell SM, Shi L, Halkowycz P and Dong Q. Structure-based design and synthesis of pyrrole derivatives as MEK inhibitors. Bioorganic and Medicinal Chemistry Letters. 2010; 20:4156-4158.

13. Wee S, Jagani Z, Xiang KX, Loo A, Dorsch M, Yao Y-M, Sellers WR, Lengauer C and Stegmeier F. PI3K Pathway Activation Mediates Resistance to MEK Inhibitors in KRAS Mutant Cancers. Cancer Research. 2009; 69:42864293.

14. Tentler JJ, Nallapareddy S, Tan AC, Spreafico A, Pitts TM, Morelli MP, Selby HM, Kachaeva MI, Flanigan SA, Kulikowski GN, Leong S, Arcaroli JJ, Messersmith WA and Eckhardt SG. Identification of Predictive Markers of Response to the MEK1/2 Inhibitor Selumetinib (AZD6244) 
in K-ras-Mutated Colorectal Cancer. Molecular Cancer Therapeutics. 2010; 9:3351-3362.

15. Spreafico A, Tentler JJ, Pitts TM, Tan AC, Gregory MA, Arcaroli JJ, Klauck PJ, McManus MC, Hansen RJ, Kim J, Micel LN, Selby HM, Newton TP, McPhillips KL, Gustafson DL, DeGregori JV, et al. Rational Combination of a MEK Inhibitor, Selumetinib, and the Wnt/Calcium Pathway Modulator, Cyclosporin A, in Preclinical Models of Colorectal Cancer. Clinical Cancer Research. 2013; 19:4149-4162.

16. Balmanno K, Chell SD, Gillings AS, Hayat S and Cook SJ. Intrinsic resistance to the MEK1/2 inhibitor AZD6244 (ARRY-142886) is associated with weak ERK1/2 signalling and/or strong PI3K signalling in colorectal cancer cell lines. International Journal of Cancer. 2009; 125:2332-2341.

17. Singh A, Greninger P, Rhodes D, Koopman L, Violette S, Bardeesy N and Settleman J. A gene expression signature associated with "K-Ras addiction" reveals regulators of EMT and tumor cell survival. Cancer Cell. 2009; 15:489500 .

18. Singh A, Sweeney MF, Yu M, Burger A, Greninger P, Benes C, Haber DA and Settleman J. TAK1 (MAP3K7) inhibition promotes apoptosis in KRAS-dependent colon cancers. Cell. 2012; 148:639-650.

19. Tentler JJ, Nallapareddy S, Tan AC, Spreafico A, Pitts TM, Morelli MP, Selby HM, Kachaeva MI, Flanigan SA, Kulikowski GN, Leong S, Arcaroli JJ, Messersmith WA and Eckhardt SG. Identification of predictive markers of response to the MEK1/2 inhibitor selumetinib (AZD6244) in K-ras-mutated colorectal cancer. Mol Cancer Ther. 2010; 9:3351-3362.

20. Pitts TM, Newton TP, Bradshaw-Pierce EL, Addison R, Arcaroli JJ, Klauck PJ, Bagby SM, Hyatt SL, Purkey A, Tentler JJ, Tan AC, Messersmith WA, Eckhardt SG and Leong S. Dual Pharmacological Targeting of the MAP Kinase and PI3K/mTOR Pathway in Preclinical Models of Colorectal Cancer. PLoS ONE. 2014; 9:e113037.

21. Flanigan SA, Pitts TM, Newton TP, Kulikowski GN, Tan AC, McManus MC, Spreafico A, Kachaeva MI, Selby HM, Tentler JJ, Eckhardt SG and Leong S. Overcoming IGF1R/ IR Resistance Through Inhibition of MEK Signaling in Colorectal Cancer Models. Clinical cancer research : an official journal of the American Association for Cancer Research. 2013; 19:6219-29. doi: 10.1158/1078-0432.CCR13-0145.

22. Yeh TC, Marsh V, Bernat BA, Ballard J, Colwell H, Evans RJ, Parry J, Smith D, Brandhuber BJ, Gross S, Marlow A, Hurley B, Lyssikatos J, Lee PA, Winkler JD, Koch K, et al. Biological Characterization of ARRY-142886 (AZD6244), a Potent, Highly Selective Mitogen-Activated Protein Kinase Kinase 1/2 Inhibitor. Clinical Cancer Research. 2007; 13:1576-1583.

23. Corcoran RB, Cheng KA, Hata AN, Faber AC, Ebi H, Coffee EM, Greninger P, Brown RD, Godfrey JT, Cohoon TJ, Song Y, Lifshits E, Hung KE, Shioda T, Dias-Santagata
D, Singh A, et al. Synthetic Lethal Interaction of Combined BCL-XL and MEK Inhibition Promotes Tumor Regressions in KRAS Mutant Cancer Models. Cancer cell. 2013; 23:121-128.

24. Tentler JJ, Tan AC, Weekes CD, Jimeno A, Leong S, Pitts TM, Arcaroli JJ, Messersmith WA and Eckhardt SG. Patient-derived tumour xenografts as models for oncology drug development. Nat Rev Clin Oncol. 2012; 9:338-350.

25. Eisenhauer EA, Therasse P, Bogaerts J, Schwartz LH, Sargent D, Ford R, Dancey J, Arbuck S, Gwyther S, Mooney M, Rubinstein L, Shankar L, Dodd L, Kaplan R, Lacombe D and Verweij J. New response evaluation criteria in solid tumours: Revised RECIST guideline (version 1.1). European Journal of Cancer. 2009; 45:228-247.

26. Troiani T, Vecchione L, Martinelli E, Capasso A, Costantino S, Ciuffreda LP, Morgillo F, Vitagliano D, D'Aiuto E, De Palma R, Tejpar S, Van Cutsem E, De Lorenzi M, Caraglia M, Berrino L and Ciardiello F. Intrinsic resistance to selumetinib, a selective inhibitor of MEK1/2, by cAMP-dependent protein kinase A activation in human lung and colorectal cancer cells. Br J Cancer. 2012; 106:1648-1659.

27. Beloueche-Babari M, Jamin Y, Arunan V, Walker-Samuel S, Revill M, Smith PD, Halliday J, Waterton JC, Barjat H, Workman P, Leach MO and Robinson SP. Acute tumour response to the MEK1/2 inhibitor selumetinib (AZD6244, ARRY-142886) evaluated by non-invasive diffusionweighted MRI. Br J Cancer. 2013; 109:1562-1569.

28. Yamaguchi T, Kakefuda R, Tajima N, Sowa Y and Sakai T. Antitumor activities of JTP-74057 (GSK1120212), a novel MEK1/2 inhibitor, on colorectal cancer cell lines in vitro and in vivo. Int J Oncol. 2011; 39:23-31.

29. Shimizu T, Tolcher AW, Papadopoulos KP, Beeram M, Rasco DW, Smith LS, Gunn S, Smetzer L, Mays TA, Kaiser B, Wick MJ, Alvarez C, Cavazos A, Mangold GL and Patnaik A. The Clinical Effect of the Dual-Targeting Strategy Involving PI3K/AKT/mTOR and RAS/MEK/ ERK Pathways in Patients with Advanced Cancer. Clinical Cancer Research. 2012; 18:2316-2325.

30. Britten C. PI3K and MEK inhibitor combinations: examining the evidence in selected tumor types. Cancer Chemotherapy and Pharmacology. 2013; 71:1395-1409.

31. Diaz Jr LA, Williams RT, Wu J, Kinde I, Hecht JR, Berlin J, Allen B, Bozic I, Reiter JG, Nowak MA, Kinzler KW, Oliner KS and Vogelstein B. The molecular evolution of acquired resistance to targeted EGFR blockade in colorectal cancers. Nature. 2012; 486:537-540.

32. Hatzivassiliou G, Haling JR, Chen H, Song K, Price S, Heald R, Hewitt JFM, Zak M, Peck A, Orr C, Merchant M, Hoeflich KP, Chan J, Luoh S-M, Anderson DJ, Ludlam $\mathrm{MJC}$, et al. Mechanism of MEK inhibition determines efficacy in mutant KRAS- versus BRAF-driven cancers. Nature. 2013; 501:232-236.

33. Gregory MA, Phang TL, Neviani P, Alvarez-Calderon F, Eide CA, O'Hare T, Zaberezhnyy V, Williams RT, Druker 
BJ, Perrotti D and Degregori J. Wnt/Ca2+/NFAT signaling maintains survival of $\mathrm{Ph}+$ leukemia cells upon inhibition of Bcr-Abl. Cancer cell. 2010; 18:74-87.

34. Davies BR, Logie A, McKay JS, Martin P, Steele S, Jenkins R, Cockerill M, Cartlidge S and Smith PD. AZD6244 (ARRY-142886), a potent inhibitor of mitogen-activated protein kinase/extracellular signal-regulated kinase kinase 1/2 kinases: mechanism of action in vivo, pharmacokinetic/ pharmacodynamic relationship, and potential for combination in preclinical models. Molecular cancer therapeutics. 2007; 6:2209-2219. 